\title{
Antiplatelets in Stroke Prevention
}

\author{
Antonio Pinto*, Domenico Di Raimondo*, Antonio Tuttolomondo ${ }^{\circ}$ and Giuseppe Licata ${ }^{\circ}$ \\ U.O.C. di Medicina Vascolare - ${ }^{\circ}$ U.O.C. di Medicina Interna e Cardioangiologia, Dipartimento Biomedico di Medicina \\ Interna e Specialistica, Università degli Studi di Palermo
}

\begin{abstract}
Stroke is the second cause of death worldwide and one of the leading causes of disability. Approximately 25\% of strokes are recurrent.

In patients who are at high risk because they already have occlusive vascular disease, long-term antiplatelet therapy (eg, with aspirin) reduces the yearly risk of serious vascular events (non-fatal myocardial infarction, non-fatal stroke, or vascular death) by about a quarter. Current recommendations for prevention of secondary stroke indicate for the broad use of antiplatelet therapy for the prevention of recurrent stroke in patients with a history of non-cardioembolic stroke or TIA.

For primary prevention, however, the balance is less clear because the risks without aspirin, and hence the absolute benefi ts of aspirin, are generally an order of magnitude lower than in secondary prevention.

In patients who are at high risk because they already have occlusive vascular disease, long-term antiplatelet therapy (e.g. with aspirin) reduces the yearly risk of serious vascular events (non-fatal myocardial infarction, non-fatal stroke, or vascular death) by about a quarter.

Although several trials have investigated the use of antiplatelet drugs in ischemic stroke patients, ascertaining the sure benefit, especially in secondary prevention in non-cardioembolic stroke, various issues remains unclarified, and new questions arise with the analysis of the results of available trials.
\end{abstract}

Keywords: ?????????????????????????

\section{INTRODUCTION}

Stroke is one of the main causes of death and disability worldwide [1]. Antithrombotic therapy is actually a cornerstone for the therapy of both acute and chronic cerebrovascular disease, having showed a clear effectiveness either in acute stroke treatment and in prevention of new cerebrovascular events. Antiplatelet agents are a heterogenous class of drugs that have been successfully used for more than 2 decades in secondary stroke prevention. These agents include aspirin, (an irreversible inhibitor of cyclooxygenase-1, which in turn inhibits the formation of tromboxane A2) with or without dipyridamole (whose mechanism of action is dominated by inhibition of platelet phosphodiesterase E5 through increasing of cyclic AMP), and more recently, the thienopyridine ticlopidine and clopidogrel (a P2Y12 adenosine diphosphate receptor blocker). Platelets play a pivotal role in the development and progression of brain ischemia. An association was observed between the extent and duration of platelet activation and stroke severity [2]. Antithrombotics prevent the formation of blood clots that can become lodged in a cerebral artery and cause strokes. Antiplatelet drugs prevent clotting by decreasing the activity of platelets, blood

\footnotetext{
*Address correspondence to this author at the Dipartimento Biomedico di Medicina Interna e Specialistica, Università degli Studi di Palermo, Palermo, Italy; Tel: ++39-091/6552102; Fax: ++39-091/6552285;

E-mail: diraimondo@unipa.it

*The two authors equally contributed to the work
}

cells that contribute to the clotting property of blood. These drugs reduce the risk of blood-clot formation, thus reducing the risk of ischemic stroke. The biological activity of antiplatelet drugs goes widely beyond the reduction of platelet activity including effects both locally on other blood elements and within the vessel wall as well as more remote effects on other cell types throughout the body. Antiplatelet drugs provide significant effects in tissues damages by cerebral ischaemia: may prevent distal and proximal propagation of arterial thrombus, prevent re-embolization, prevent platelet aggregation in the microcirculation, reduce the release of thromboxane and other neurotoxic eicosanoids. All these effects in the acute phase guarantee a reduction of acute stroke severity, through reduction of neuronal damage, and therefore, a more favourable outcome.

The importance of antiplatelet agents for both the prevention and treatment of ischemic disease was a concept developed as the consequence of some research that showed: (a) the contribution of platelets to both cardiac [3] and carotid disease; (b) development of reproducible assays to quantify platelet activation [4].

Most strokes are caused by a sudden blockage of an artery in the brain (called an ischaemic stroke) that is usually due to a blood clot. Treatment with antiplatelet drugs such as aspirin may prevent clots from forming preventing ischemic event and, during the acute phase of stroke, reducing formation of new clots, hence improve recovery. However antiplatelet drugs may also cause bleeding in the brain which 
could offset any benefits. Several studies have evaluated the role of one antiplatelet agent, aspirin, in reducing stroke severity [5-7]. Mechanisms by which antiplatelet agents may reduce stroke severity include stabilization of plaque and decreasing platelet aggregation thereby reducing size and frequency of thromboembolic emboli [8]. Furthermore, it is thought that antiplatelet agents such as aspirin may confer additional benefit on stroke severity beyond their presumed primary mechanism of action through anti-inflammatory and neuroprotective mechanisms [9].

Aim of this review is to summarize the main available evidence regarding the use of antiplatelet drugs in both primary and secondary prevention of ischemic stroke.

\section{ANTIPLATELET DRUGS FOR PRIMARY PREVEN- TION OF ISCHEMIC STROKE}

The strategy of prevention of the first ischemic cerebral event represent a decisive approach in order to reduce morbility and mortality related to cerebrovascular disease. We have consistent evidence that a global intervention aimed to reduce the burder of risk of the patient is a winning approach for reducing cereborvascular risk. So, tight control of hard risk factor such as hypertension or the prevention or reduction of other target-organ damage, including congestive heart failure and renal failure are of great effectiveness [10].

Overall, antihypertensive therapy is associated with a $35 \%$ to $44 \%$ reduction in the incidence of stroke [11]. Likewise, statin therapy has been shown to produce consistent benefits in large randomized trials in decreasing stroke risk [12]. The risk of stroke in diabetic patients with hypertension is increased and blood pressure and blood glucose levels should be controlled. Atrial fibrillation alone is associated with a 3- to 4-fold increased risk of stroke after adjustment for other vascular risk factors [13].

Over the control of cerebrovascular risk factor, effectiveness of treatment with antiplatelet drugs of relatively healthy but high-risk subjects, in order to prevent the first ischemic event was tested in several trials.

\section{ASPIRIN}

Aspirin is surely the most widely studied antiplatelet drug, and is considered affordable, broadly available, and reasonably safe. Data collected in three decades of trials attributed to aspirin a potential combined risk reduction of stroke, myocardial infarction and vascular death by a $23 \%$ [14]. Clinical benefits over placebo have been shown for doses from $50 \mathrm{mg}$ to $1.3 \mathrm{~g}$, but the risk of upper gastrointestinal events (ulcers or bleeding) increases with the dose, so that some recommendations insist that 'doses greater than 80 mg should not be routinely prescribed, whereas a larger spectrum (50-325 mg) is accepted in other recommendations

Although aspirin increases the risk of major bleeding by about $70 \%$ (risk ratio [RR] 1.71 [95\% CI 1.41-2.08]), the absolute annual increase is modest (0.13\% [0.08-0.20\%]), indicating that one additional major bleeding episode will occur each year for every 769 patients (95\% CI 500-1250) treated with aspirin $[15,16]$.

The Physicians' Health Study [17], was a randomized, double blind, placebo-controlled trial designed to determine whether low-dose aspirin (325 mg every other day) decreased cardiovascular mortality. Among 22,071 participants with a mean follow-up of 60.2 months, although a $44 \%$ RRR of myocardial infarction, there was a slighty unsignificant increase of risk of stroke, mainly due to hemorrhagic stroke. As a result, this study did not advocate for aspirin as a primary preventive strategy for stroke.

The Women's Health Study [18], randomized 39,876 initially asymptomatic women aged 45 years of age and older to receive $100 \mathrm{mg}$ of aspirin or placebo on alternate days. Patients were followed for 10 years, with the primary endpoint being a first major vascular event (non-fatal MI, non-fatal stroke, or cardiovascular death). Unlike data from prior studies that included mainly men, this study found a non-significant 9\% reduction (Relative Risk $(\mathrm{RR})=0.91$; 95\% Confidence Interval (CI) 0.80 to $1.03 ; \mathrm{P}=0.13$ ) in the combined primary end point among women, but a statistically significant $17 \%$ reduction in the risk of stroke $(\mathrm{RR}=$ $0.83 ; 95 \%$ CI 0.69 to $0.99 ; \mathrm{P}=0.04)$. This was based on a $24 \%$ reduction in the risk of ischemic stroke and a nonsignificant increase in the risk of hemorrhagic stroke. The most consistent benefit for aspirin was in women $\geq 65$ years of age at study entry.

Recently, a new Antithrombotic Trialists (ATT) metaanalyses [19] evaluated incidence of serious vascular events (myocardial infarction, stroke, or vascular death) and major bleedings in six primary prevention trials $(95,000$ individuals at low average risk, 660,000 person-years, 3554 serious vascular events) and 16 secondary prevention trials $(17,000$ individuals at high average risk, 43,000 person-years, 3306 serious vascular events) that compared long-term aspirin vs. control. In the primary prevention trials, aspirin allocation yielded a $12 \%$ proportional reduction in serious vascular events $(0.51 \%$ aspirin $v s$. $0.57 \%$ control per year, $\mathrm{p}=0.0001)$, due mainly to a reduction of about a fifth in non-fatal myocardial infarction $(0.18 \%$ vs. $0.23 \%$ per year, $\mathrm{p}<0.0001)$. The net effect on stroke was not significant $(0.20 \%$ vs. $0.21 \%$ per year, $\mathrm{p}=0.4$ : haemorrhagic stroke $0.04 \%$ vs. $0.03 \%, \mathrm{p}=0.05$; other stroke $0.16 \%$ vs. $0.18 \%$ per year, $\mathrm{p}=0.08)$. Vascular mortality did not differ significantly $(0.19 \%$ vs. $0.19 \%$ per year, $\mathrm{p}=0.7)$. Aspirin allocation increased major gastrointestinal and extracranial bleeds $(0.10 \%$ vs. $0.07 \%$ per year, $\mathrm{p}<0.0001)$, and the main risk factors for coronary disease were also risk factors for bleeding. The data collected regarding the aggregate of all serious vascular events seemed similar for men and women. On the basis of this new ATT metanalysis authors conclude that in primary prevention without previous disease, aspirin is of uncertain net value as the reduction in occlusive events needs to be weighed against any increase in major bleeds. Further trials are in progress.

The Chinese Acute Stroke Trial (CAST) [20], and the concurrent International Stroke Trial (IST) [21], tested a policy of start aspirin immediately when a cerebral ischdmia is suspected; so aspirin should be started as soon as a CT or MR scan has been performed and has excluded intracranial haemorrhage as the cause of the stroke. Results of these two big trials shows clearly that starting daily aspirin promptly in patients with suspected acute ischemic stroke also reduces the immediate risk of further stroke or death in hospital and the overall risk of death or dependency, concluding that early aspirin is of benefit for a wide range of patients, and its 
prompt use should be routinely considered for all patients with suspected acute ischemic stroke, mainly to reduce the risk of early recurrence. However, some uncertainly remains about the effects of early aspirin in particular categories of patient with acute stroke (eg, the elderly, those without a CT scan, or those with atrial fibrillation).

According to the European Stroke Organisation (ESO) Guidelines for Management of Ischaemic Stroke and Transient Ischaemic Attack [22] the use of low dose ASA in primary prevention is recommended in women aged 45 years or more who are not at increased risk for intracerebral haemorrhage and who have good gastrointestinal tolerance nevertheless cklincial studies have showed no dramatical preventive effect. It is also recommended that low-dose ASA may be considered in men for the primary prevention of MI; however, it does not reduce the risk of ischaemic stroke. ASA may be recommended for patients with non-valvular AF who are younger than 65 years and free of vascular risk factors. Unless contraindicated, either ASA or an oral anticoagulant (international normalized ratio, INR, 2.0-3.0) is recommended for patients with non-valvular AF who are aged $65-$ 75 years and free of vascular risk factors. Low-dose ASA is recommended also for patients with asymptomatic ICA stenosis $>50 \%$ to reduce their risk of vascular events. Other antiplatelet drugs than ASA have not been studied in asymptomatic subjects and therefore cannot be recommended for primary stroke prevention.

\section{CLOPIDOGREL}

Clopidogrel is a thienopyridine which has largely replaced ticlopidine because of its much better hematologic and cutaneous safety. Clopidogrel is an inactive prodrug that requires two-step oxidation by the hepatic cytochrome $\mathrm{P} 450$ (CYP) system to generate its active compound, the thiol metabolite, which targets and irreversibly inhibits the ADP P2Y purinoceptor 12 on circulating platelets. The hepatic CYP isoenzymes involved in this two-step metabolisation process of clopidogrel include CYP2C19, CYP3A4/5, CYP1A2, CYP2B6, and CYP2C9.

Several trial addressed the issue of the comparative effect between clopidogrel and aspirin in prevention of stroke.

In the CHARISMA Study [23] a prospective, multicenter, randomized, double-blind, placebo-controlled study, 15,603 patients were randomized to receive clopidogrel 75 $\mathrm{mg}$ /day plus lowdose aspirin (75-162 mg/day) or placebo plus low-dose aspirin, with median follow-up of 28 months. All patients were 45 years of age or older and had either multiple atherothrombotic risk factors or a history of documented coronary disease, cerebrovascular disease, or symptomatic PAD. The results showed no statistically significant difference between treatment groups in the rates of occurrence of the primary efficacy endpoint (clopidogrel plus aspirin $6.8 \%$, aspirin alone $7.3 \%$; RR 0.93 , 95\% CI 0.83-1.05; $\mathrm{p}=0.22$ ). Patients with multiple risk factors but no clearly established vascular disease (primary prevention cohort) did not benefit from the addition of clopidogrel to aspirin; instead, adjunctive clopidogrel was associated with a nonsignificant $20 \%$ relative increase in the rate of primary events, as well as an excess in cardiovascular mortality (3.9 vs. $2.2 \%, \mathrm{p}=0.01)$.
A recent Cochrane review [24] found, evaluating 19.185 high risk vascular patients, a significantl but strictly marginal improvement of long-term reduction of risk of stroke and other major vascular events by $8.7 \%$ (95\% CI $0.3-16.5 \%)$ through use of clopidogrel in comparison to aspirin. Clopidogrel also causes less gastrointestinal bleeding than $325 \mathrm{mg}$ aspirin daily (RR 0.69 [95\% CI 0.48-1.00]; absolute annual decrease $0.12 \%$ [0.00-0.28\%]), but does not reduce the risk of other types of bleeding. However, the cost of clopidogrel is substantially greater than that of aspirin, and this might prevent its use, especially in less developed countries [24].

\section{ANTIPLATELET DRUGS FOR SECONDARY PRE- VENTION OF ISCHEMIC STROKE}

\section{Approximately $25 \%$ of Strokes are Recurrent}

Patients experiencing stroke or transient ischemic attack (TIA) are at high risk for recurrent (secondary) strokes, which comprise $29 \%$ of all strokes in the United States. Prospective studies have shown that the early risk of stroke post TIA is much higher than previously thought $-18 \%$ at 90 days. More importantly, most of the recurrent strokes happened within the first 2 days after the index event [25, 26]. After 1 year the risk falls at about 5\% per year [27].

Current recommendations for prevention of secondary stroke from the American College of Chest Physicians (ACCP) [28] indicates for the broad use of antiplatelet therapy for the prevention of recurrent stroke in patients with a history of non-cardioembolic stroke or TIA. Five agentsaspirin, ticlopidine, clopidogrel, extended-release dipyridamole (ER-DP), and triflusal--have demonstrated efficacy in large-scale clinical studies in the prevention of recurrent vascular events and/or stroke in patients with a history of stroke.

Although clinicians may choose acetylsalicylic acid (ASA) as first-line therapy for secondary prevention, clinical guidelines and evidence from trials suggest that ASA may not be the most effective strategy [29].

\section{ASPIRIN}

Aspirin represents an important therapeutic regimen to prevent the recurring of ischemic events or vascular death after an ischemic stroke.

The antiplatelet efficacy of aspirin in preventing secondary stroke was established by three studies conducted in the late 1980 s and early 1990s. The first of three was the Swedish Aspirin Low-dose (SALT) Trial [30] that have demonstrated that aspirin, even in doses as low as $30 \mathrm{mg} /$ day, reduces secondary stroke, Myocardial infarction, or vascular death in patients with previous vascular event. At a dosage of $75 \mathrm{mg} /$ day in this trial aspirin reduced the rate of recurrent stroke by $18 \%$.

Two subsequent studies, the Stroke Prevention in Reversible Ischemia Trial (SPIRIT) [31], an European trial that compared warfarin with aspirin in patients with TIA or minor stroke during the previous six months, and the Warfarin vs. Aspirin in Recurrent Stroke Prevention Study (WARSS) [32], in which lower doses of warfarin were hypothesize to guarantee a superior benefit to aspirin without the risk of untoward outcomes observed in the SPIRIT trial [31], 
showed that aspirin was preferable to warfarin in preventing secondary stroke in patients with initial non-cardioembolic stroke.

Given the results of these studies, was clear that aspirin should be the therapeutic choice for secondary stroke prevention unless a clear cardioembolic source, such as atrial fibrillation, is present.

The fact that low dose aspirin is of substantial benefit for people who already suffered of occlusive vascular disease was recently confirmed by ATT Collaboration Meta-analysis [19]: in the secondary prevention trials, aspirin allocation yielded a greater absolute reduction in serious vascular events $(6.7 \%$ vs. $8.2 \%$ per year, $\mathrm{p}<0.0001)$, with a nonsignificant increase in haemorrhagic stroke but reductions of about a fifth in total stroke $(2.08 \%$ vs. $2.54 \%$ per year, $\mathrm{p}=0.002)$ and in coronary events $(4.3 \%$ vs. $5.3 \%$ per year, $\mathrm{p}<0.0001)$

Despite the fact that ASA remains the most popular, most commonly prescribed and widely accepted agent for secondary stroke prevention in the world, its efficacy still does not exceed $25 \%$. The development of the benchmark techniques for platelet-mediated haemostasis and platelet function monitoring in ASA users raise the possibility to triage low response or so-called "ASA resistance". The incidence of clinical aspirin resistance varies from $11 \%$ to $17.3 \%$ depending on the definition adopted [33], whereas the incidence of biochemical resistance ranges from as little as $5 \%$ to as much as $60 \%$ depending primarily on the platelet function assay used [34]. A high prevalence of ASA resistance (28.0$65.0 \%$ ) was also demonstrated among post-stroke ASA users [35]. None of the available platelet aggregation assays have convincingly been shown to correlate with recurrence of clinical events (ischemic stroke or MI) and the results between different assays have also been discordant. Because Aspirin resistance is a poorly defined phenomenon and it is likely to be multifactorial, and, to date, no standardized definition or test can be used to quantify either type of aspirin resistance as well as do not exist a therapeutic algorithm that helps clinical to evaluate how to treat an aspirin-resistant patient.

The association between increased platelet activity and high blood lipid content is not new. In an early small retrospective clinical study, hyperlipidemia has been found to enhance the risk of ASA failure in patients after first-ever ischemic stroke [36]. Indeed, the prevalence of hyperlipidemia in the ASA-resistant patients in that study was $65 \%$, compared to $26 \%$ of the average ASA responders. A slight correlation between ASA resistance and conventional lipid biomarkers has been found in another study [37]. Patients with ASA resistance exhibited a higher total cholesterol/ HDL ratio and lipoproteins compared to ASA responders.

The assessment of the extent of adenosine diposphate (ADP)-induced platelet aggregation and its correlation with the outcome after an acute ischemic stroke in patients already taking aspirin therapy was the aim of a study by Cha et al. [38]. This study underlined individual differences in subjects taking aspirin and assessed through different degree of ADP-induced platelet aggregation: increased ADP-induced aggregation is associated with a poor outcome after an acute ischemic stroke. This confirms, once more, a constitutional different degree of aspririn activity from a subject to another.

\section{TICLOPIDINE}

Ticlopidine belongs to the group of thienopyridines, whose antiplatelet effect is based on the inhibition of adenosindiphosphate (ADP) and also on the blockage of the ADP mediated binding of fibrinogen membrane thrombocyte receptor, glycoprotein IIb/IIIa.

The Ticlopidine Aspirin Stroke Study (TASS) [39] found only slightly higher efficacy of ticlopidine $250 \mathrm{mg}$ twice daily than ASA $1300 \mathrm{mg}$ once daily in the secondary prevention of stroke. However, when compared to ASA, the use of ticlopidine is associated with markedly more frequent side effects, such as diarrhea, gastro-intestinal intolerance, skin rash and haematological disorders but not with increased bleeding complications. Owing to these severe side effects ticlopidine is usually used as a second choice antiplatelet drug reserved for patients with ASA intolerance.

\section{CLOPIDOGREL AND THE COMBINATION ASPIRIN + CLOPIDOGREL}

Combined antiplatelet agents may offer additive protection over single drugs after stroke. Clopidogrel has been evaluated as monotherapy and in combination with aspirin with regard to its efficacy in preventing secondary stroke.

In the Clopidogrel vs. Aspirin in Patients at Risk of Ischemic Events (CAPRIE) study [40], three groups with a recent history of symptomatic cardiovascular disease (stroke, MI, or peripheral arterial disease) were randomized to clopidogrel $75 \mathrm{mg} /$ day or aspirin $325 \mathrm{mg} / \mathrm{day}$ to evaluate the composite outcome of ischemic stroke, MI, or vascular death as well as the relative safety of each drug. Clopidogrel was slightly more effective than aspirin in decreasing the combined risk of ischemic stroke, myocardial infarction, and vascular death, in patients with symptomatic atherosclerotic vascular disease (8.7\% Relative Risk Reduction; $p=0.043$ in intention to treat and $9.4 \%$ in an on-treatment analysis). However, clopidogrel did not demonstrate superiority vs. aspirin in preventing recurrent stroke among patients with a history of stroke ( $8 \%$ RRR; $p=0.28)$, although the study was powered only to demonstrate significant differences in the overall population $(n=19,185)$. No major safety differences were observed between clopidogrel and aspirin, although the rate of serious hemorrhage was slightly higher in the aspirin group (1.55 vs. 1.38\%); cutaneous rashes and diarrhea were more frequent with clopidogrel [40].

In the MATCH Trial [41] on the basis of previous trial results (including CAPRIE) in patients with cardiac and cerebrovascular disease, investigators sought to determine whether the addition of aspirin to clopidogrel would further reduce the risk of recurrent ischemic attacks in high-risk patients after recent ischemic stroke or TIA. Patients were included if they had a stroke or TIA within the previous 3 months and 1 or more of 5 additional high-risk factors within the previous 3 years: previous stroke, previous MI, angina, diabetes, or symptomatic PAD. The results of MATCH showed no significant difference between clopidogrel alone and clopidogrel plus aspirin in reducing risk of vascular 
events after stroke or TIA. Although there was an absolute risk reduction of $1 \%$ and a relative risk reduction of $6.4 \%$ favoring clopidogrel plus aspirin, the between-group differences were not statistically significant. Should be underlined, however, that there was a beneficial trend of the association antiplatelet therapy in the subgroup of patients randomly assigned to treatment within 7 days of a TIA or stroke.

Furthermore, in the CHARISMA Study Study [23], previously described in this review, analysis of data provided by patients with established cardiovascular disease (the secondary prevention cohort), showed that the addition of clopidogrel to aspirin therapy resulted in a marginally significant clinical benefit regarding the primary endpoint (6.9 vs. $7.9 \%$ with placebo; RR 0.88; 95\% CI, 0.77-0.998; p = 0.046). Results of the safety analysis showed a non-significant increase in the primary safety endpoint of severe bleeding with clopidogrel; the rate of moderate bleeding (that required transfusion) was $2.1 \%$ in the clopidogrel group and $1.3 \%$ in the placebo group (RR, 1.62; 95\% CI, 1.27-2.08; p<0.001).

Assessment of degree of platelet inhibition using one antiplatelet agent or the combination of two was the aim of a study involving 31 subjects with previous atherothrombotic or lacunar stroke. Patients were treated with aspirin (100 to $300 \mathrm{mg} / \mathrm{d})$, clopidogrel $(75 \mathrm{mg} / \mathrm{d})$ or both aspirin and clopidogrel for 4 weeks [42]. Using as platelet-activation criterion collagene/ADP closure time authors demonstrated an higher degree of protection under combination therapy of aspirin + clopidogrel. Obviuosly, a lower risk of thrombisis is ever associated to a higher hemorrhagic risk.

Given the results showed, clopidogrel has been increasingly used in secondary prevention after an ischemic stroke. Further data regarding the topic whether therapy with clopidogrel + aspirin $(\mathrm{C}+\mathrm{ASA})$ will produce more evident platelet inhibition than aspirin alone (ASA) in patients after ischemic stroke were provided by Serebruany et al. in the PLUTO Trial [43]. In this study therapy with C+ASA resulted in a significant inhibition of platelet activity assessed by ADP$(\mathrm{P}=0.00001)$ and collagen-induced $(\mathrm{P}=0.02)$ aggregation; closure time prolongation $(\mathrm{P}=0.03)$, and reduction of platelet activation units, indicating a greater inhibition of platelet activity of combination therapy than ASA alone in patients after an ischemic stroke.

The question of ultra-early secondary prevention, however, remains open, given the 1/3 relative risk reduction observed in the Fast Assessment of Stroke and Transient Ischemic Attack to Prevent Early Recurrence (FASTER) study [44]. Patients with transient ischaemic attack (TIA) or minor stroke are at high immediate risk of stroke. The optimum early treatment options for these patients are not still well assessed. Authors in this work ascertained that aggressive and precocious secondary prevention operated adding clopidogrel to aspirin that was already used by all the subjects enrolled, was able to provide a reduction of recurrence of ischemic events. The question of aggressive secondary prevention using combination therapy of antiplatelet drugs merits further studies.

On the basis of the current evidence for long-term treatment of survivors of ischemic stroke or TIA, clopidogrel is an effective alternative for patients who are intolerant to aspirin [44-46].
Likewise aspirin, a so called clopidogrel-reistance has been identificated in the latest years, to individuate the cause of the occurrence in a variable amount of patients treated with a dequate dosage of clopidogrel of ischemic of ischemic events.

The prevalence of clopidogrel non-response in different populations has been described as between $4 \%$ and $30 \%$. There are several potential mechanisms of clopidogrel resistance, which can be divided into two main groups, both involving CYP metabolism: 1) extrinsic mechanisms (i.e. drug interactions involving CYP3A4) and 2) intrinsic mechanisms (i.e. polymorphisms of $\mathrm{P} 2 \mathrm{Y} 12$ receptor and CYP3As). In regard to drug interactions, any medication that interacts with (either inhibiting or increasing) CYP3A4 can potentially block the conversion of clopidogrel into its active metabolite. Among these drugs, statins, except for pravastatin, might interfere with clopidogrel metabolism. The antiplatelet activity has also been shown to be reduced in about a third of patients receiving omeprazole, protonpump Inhibitor which is a CYP2C19 inhibitor [47]. However, other authors have critically evaluated this observation [48].

Recently, several reports indicate that certain polymorphisms in the hepatic cytochrome p450 system are associated with an excess of vascular events. Patients who are carriers of a loss of function of CYP2C19 allele (including the $* 2$ and $* 3$ alleles) might have a reduced rate in the conversion to an active metabolite, therefore resulting in a decreased inhibition of platelets. Carriers of less functional alleles compared with non-carriers showed a significant increase in the risk of major vascular events $[49,50]$. A recent analysis of data among 5,059 patients with acute coronary syndromes or atrial fibrillation showed that the response to clopidogrel compared with placebo was consistent, irrespective of CYP2C19 loss-of-function carrier status [51]. Therefore, it is possible that loss-of-function variants do not directly alter the efficacy and safety of clopidogrel. Consequently, clopidogrel should be used regardless of the carrier status until further studies can elucidate this paradox.

\section{DIPYRIDAMOLE AND THE COMBINATION ASPI- RIN + DIPYRIDAMOLE}

Dipyridamole is a weak antiplatelet drug that has been combined with aspirin for over 30 years in the secondary prevention of non-cardioembolic ischemic stroke. By contrast with four early studies, which showed no benefit of the combination over aspirin alone using immediate-release dipyridamole formulation, more recent studies using extended-release dipyridamole and higher doses (400 mg), such as the European Stroke Prevention Study 2 (ESPS2) [52], one of two studies that evaluated aspirin plus extendedrelease dipyridamole for secondary stroke prevention, showed a significant benefit of the combination over aspirin alone $(50 \mathrm{mg})$. In ESPS-2, 6,602 patients who had a recent ischemic stroke or TIA were enrolled in a multicenter, double-blind, placebo-controlled trial that randomly assigned them to one of four treatment groups: aspirin $(25 \mathrm{mg}$, twice daily), extended-release dipyridamole (200 mg, twice daily), aspirin plus extended-release dipyridamole, or placebo. Both agents given as monotherapy demonstrated an independent and statistically significant reduction in recurrent stroke (18\% [P $=0.013]$, and $16 \%,[\mathrm{P}=0.039]$, respectively). How- 
ever, the combination of aspirin plus extended-release dipyridamole reduced stroke recurrence by $23.1 \%(\mathrm{P}=$ $0.006)$ compared with aspirin alone and by $37 \%(\mathrm{P}<0.001)$, compared with placebo. Results from ESPS-2 indicate that aspirin plus extended-release dipyridamole has significant benefit over aspirin alone for prevention of second stroke. The benefit of the combination was obtained without a significant increase in hemorrhagic events.

The European/Australasian Stroke Prevention in Reversible Ischaemia (ESPRIT) Study Group [53] included a meta-analysis of six comparative trials, including a total of 3,888 patients taking aspirin plus dipyridamole and 3,907 taking aspirin alone; this analysis demonstrated an overall RRR for combination therapy vs. aspirin of $18 \%$ (95\% CI, 0.74-0.91) for the composite outcome of vascular death, non-fatal stroke, or non-fatal MI. ESPRIT investigators found that combination treatment was not associated with a higher complication rate than ASA monotherapy, but that the rate of withdrawal due to adverse events was higher in the group that received the combination.

The Prevention Regimen for Effectively avoiding Second Strokes (PROFESS) trial [54] was initially designed to compare 2 combinations: aspirin + dipyridamole and aspirin + clopidogrel, but aspirin was stopped in the aspirin +clopidogrel arm after the results of the MATCH trial [41] when 1,920 patients had been included. PROFESS is thus mostly a comparison of the combination aspirin $+\mathrm{ER}$ dipyridamole $(25 \mathrm{mg}+200 \mathrm{mg}$ twice daily) and clopidogrel (75 mg daily) alone. It included 20,332 high-risk patients with a mean age of 66.1 years, less than 90-120 days after a TIA or ischemic stroke. After a median follow-up of 2.4 years, recurrent stroke occurred in $9 \%$ of patients in both groups $(9.0 \%$ on aspirin plus ER dipyridamole $v s .8 .8 \%$ on clopidogrel; HR 1.01 [95\% CI 0.92-1.11]), and the composite outcome of stroke, myocardial infarction and death from vascular causes occurred in $13.1 \%$ of patients in both groups. Thus, the combination of aspirin + ER dipyridamole is not better than clopidogrel alone in preventing stroke recurrence.

Patients with transient ischaemic attacks (TIA) and minor ischaemic strokes are at risk of serious vascular events (death from all vascular causes, non-fatal stroke, or non-fatal myocardial infarction). Their risk of vascular events lies between 4 and 11 percent per year. The combination of aspirin and ER dipyridamole is significantly more effective than aspirin alone in reducing the risk of stroke and other major vascular events (hazard ratio [HR] 0.82 [95\% CI 0.72-0.92]), without excessive bleeding or myocardial infarction in patients with previous TIA or ischaemic stroke. Aspirin only, in a daily dose of $30 \mathrm{mg}$ or more, offers only modest protection in such patients: it reduces the incidence of major vascular events by 13 percent. Adding ER dipyridamole to aspirin was associated with a 22 percent reduction in the risk of major vascular events compared with aspirin alone [55].

\section{CONCLUSIONS}

Although the certain clinical value of antiplatelet therapy, and especially of aspirin, is well recognized, several questions about antiplatelet therapy in subject with ischemic stroke remain open and need to be further clarified; first: effectiveness of combination therapy with anticoagulants. Anticoagulant therapy in ischemic stroke is the matter of another review in this journal, but in the 2002 a Cochrane review [56] and a recent critical review [57] showed that combining antiplatelet and anticoagulant therapy appears to be more effective than antiplatelet therapy alone for the initial and longterm management of ACS, and is more effective than anticoagulants alone in patients with mechanical heart valves, but increases the risk of bleeding. There is no evidence from RCTs that combination therapy compared with antiplatelet therapy alone is more effective for the prevention of recurrent cardiovascular events in patients with noncardioembolic stroke or peripheral artery disease, or compared with anticoagulant therapy alone for the prevention of stroke in patients with atrial fibrillation. Combination therapy is commonly used in patients with separate indications for antiplatelet therapy (e.g. acute coronary syndrome, recent coronary artery stent) and anticoagulant therapy (e.g. atrial fibrillation with at least one additional risk factor for stroke) despite lack of evidence from randomized controlled trials.

Second: timing of antiplatelet administration. Third: choose of antiplatelet drug and effecfiveness of combination therapy in different clinical settings. This matter has been yet investigated in several trial, analyzed previously in this review; summarizing the available evidences, the indirect comparisons of different agents seems not to show any evidence of significant heterogeneity of effect between the different agents tested: aspirin alone, ticlopidine alone, the combination of aspirin and dipyridamole, thromboxane synthase inhibitor, and the GP IIb/IIIa inhibitor abciximab. In comparative monotherapy studies of patients with previous stroke, ticlopidine seems to demonstrate slight statistically significant improved efficacy over aspirin, and clopidogrel demonstrates non-significant slight improvement over aspirin for the prevention of ischemic cardiac and cerebrovascular events; however, the adverse event profile of ticlopidine (including rash, diarrhea, and neutropenia) will probably limit its long-term use. Among combination approaches, only aspirin plus ER-DP has demonstrated statistically significant, clinically meaningful additive benefit over monotherapy with each agent.

Fourth: optimal dosage of antiplatelet drugs. The benefits of aspirin in acute stroke are drawn from trials which tested a dose of aspirin between $160 \mathrm{mg}$ and $330 \mathrm{mg}$ daily. In acute myocardial infarction, $160 \mathrm{mg}$ is the lowest dose that has been shown to be effective [19]. Lower doses of aspirin are effective for long-term secondary stroke prevention, but have not been evaluated in acute stroke. There is some (but not abundant) evidence that at least $120 \mathrm{mg}$ of aspirin is needed to acetylate all circulating platelets within a short period of time [20]. Fifth: way of administration. For patients who can swallow, aspirin can be given by mouth. However, as many people with stroke are unable to swallow, another route may need to be used on occasions. In the CAST trial [20], aspirin was given via a nasogastric tube, and in the IST [21], as a rectal suppository, or intravenously as $100 \mathrm{mg}$ of the lysine salt of acetylsalicylic acid.

Sixth: do the future provide new antiplatelet compunds, actually under development, able to offer addictional benefit without increase the risk of bleedings? 
Other interesting topics regards, for example, the possibility of different degree of activity of antiplatelet drugs when used in combination with other class of drugs such as antihypertensives, namely angiotensin-II blocker agents and statins. About this, Yu et al. recently reported a more favorable functional outcome ten days after an acute stroke in subjects taking the combination of these three classes of therapies [58]. Furthermore, a putative neuroprotective action of antiplatelet compounds and of other class of drugs such as angiotensin receptor blockers (ARBs) was hypothesized. In the PRoFESS Trial the combination aspirin + dypidiridamole, the thyenopiridine clopidogrel and the ARB telmisartan were tested, among the other oucomes, also for the assessment of this effect. Unfortunately, there was no significant difference in the proportion of patients with recurrent stroke with a good outcome, as measured with the Barthel index, across all treatment groups. Additionally, there was no significant difference in the median minimental state examination (MMSE) scores, and the number of patients with dementia among the treatment groups. Disability due to recurrent stroke and cognitive decline in patients with ischaemic stroke were not different between the two antiplatelet regimens and were not affected by the preventive use of telmisartan.

As underlined before, antiplatelet medications are the agents of choice for secondary prevention of non-cardioembolic ischemic strokes. In patients who are at high risk because they already have occlusive vascular disease, longterm antiplatelet therapy (e.g. with aspirin) reduces the yearly risk of serious vascular events (non-fatal myocardial infarction, non-fatal stroke, or vascular death) by about a quarter. Multiple clinical trials have proven their reliable albeit modest clinical benefits and relatively good safety profile. This decrease typically corresponds to an absolute reduction of about 10-20 per 1000 in the yearly incidence of non-fatal events, and to a smaller, but still definite, reduction in vascular death. Against this benefit, the absolute increase in major gastrointestinal or other major extracranial bleeds is an order of magnitude smaller. Hence, for secondary prevention, the benefits of antiplatelet therapy substantially exceed the risks.

For primary prevention, however, the balance is less clear because the risks without aspirin, and hence the absolute benefi ts of aspirin, are generally an order of magnitude lower than in secondary prevention. Previous meta-analyses of primary prevention trials were not based on individual participant data, so they could not compare reliably the benefits and risks of aspirin in prognostically important groups (such as older people and others at increased risk of coronary heart disease), and could not quantify reliably the extent to which people at increased risk of coronary heart disease might also be at increased risk of bleeding.

The most commonly recommended antiplatelet agents for secondary stroke prevention in North America and Europe are aspirin, clopidogrel, and the combination of aspirin and extended-release dipyridamole. Because of the multiple pharmacologic mechanisms available for platelet inhibition, combination antiplatelet agents have the potential for synergistic effects. However, combinations of antithrombotic agents do not necessarily improve clinical efficacy and are typically associated with increased toxicity. Clopidogrel and aspirin have been used in combination in patients with diverse arterial vascular diseases. Combination antiplatelet therapy with clopidogrel and aspirin has established clinical benefits, particularly in coronary disease and in patients who have undergone coronary stenting. Although it is tempting to extrapolate the benefits of clopidogrel and aspirin to the setting of secondary stroke prevention, recent clinical trials have failed to document significant clinical benefits in cerebrovascular patients. This failure has occurred because of a lack of significant efficacy for prevention of vascular events and a substantial increase in bleeding risk. Therefore, the clopidogrel and aspirin combination is not recommended for recurrent stroke prevention. In general, when clopidogrel is used for cerebrovascular patients, the addition of aspirin should be avoided unless there is a specific cardiac indication such as recent acute coronary syndrome or a coronary stent.

The use of the combination therapy of aspirin + ER-DP is supported by Class I data from two large studies demonstrating superiority over aspirin alone for recurrent stroke prevention. Although dual antiplatelet therapy with clopidogrel and aspirin has never been directly compared with the combination of aspirin and extended-release dipyridamole, clinical trial results favor the latter for secondary stroke prevention. Currently, there are no data for primary stroke prevention with dual antiplatelet agents regarding aspirin and extendedrelease dipyridamole. Limited data from the recent Clopidogrel for High Atherothrombotic Risk and Ischemic Stabilization Management and Avoidance (CHARISMA) Study [23] trial indicate that the combination of clopidogrel and aspirin may be harmful, compared with aspirin alone.

\section{CONFLICT OF INTEREST}

The authors confirm that this article content has no conflicts of interest.

\section{ACKNOWLEDGEMENTS}

Declared none.

\section{REFERENCES}

[1] Donnan GA, Fisher M, Macleod M, Davis SM. Stroke. Lancet 2008; 371: 1612-23.

[2] Yi HK, Liou CW, Chang HW, Lan MY, Liu JS, Chen MC. Link between platelet activity and outcomes after an ischemic stroke. Cerebrovasc Dis 2005; 20: 120-8.

[3] Badimon L, Badimon JJ. Mechanism of arterial thrombosis in nonparallel streamlines: platelet growth at the apex of stenotic severely injured vessel wall: experimental study in the pig model. J Clin Invest 1989; 84: 1134-44.

[4] Fisher CM. Concerning recurrent transient cerebral ischemic attacks. Can Med Assoc. 1962; 86: 1091-109.

[5] Wilterdink JL, Bendixen B, Adams HP, Jr, et al. Effect of prior aspirin use on stroke severity in the Trial of Org 10172 in Acute Stroke Treatment (TOAST). Stroke 2001; 32: 2836-40.

[6] Grotta J, Lemak N, Gary H, Fields W, Vital D. Does platelet antiaggregant therapy lessen the severity of stroke? Neurology 1985; 35: 632-6.

[7] De Keyser J, Herroelen L, De Klippel N. Early outcome in acute ischemic stroke is not influenced by the prophylactic use of lowdose aspirin. J Neurol Sci 1997; 145: 93-6.

[8] Arrebola MM, De la Cruz JP, Villalobos MA, et al. In vitro effects of clopidogrel on the platelet-subendothelium interaction, platelet 
thromboxane and endothelial prostacyclin production, and nitric oxide synthesis. J Cardiovasc Pharmacol 2004; 43: 74-82.

[9] Grilli M, Pizzi M, Memo M, Spano P. Neuroprotection by aspirin and sodium salicylate through blockade of nf-kappab activation. Science 1996; 274: 1383-5.

[10] Chobanian AV, Bakris GL, Black HR, et al.; National Heart, Lung, and Blood Institute Joint National Committee on Prevention, Detection, Evaluation, and Treatment of High Blood Pressure; National High Blood Pressure Education Program Coordinating Committee. The Seventh Report of the Joint National Committee on Prevention, Detection, Evaluation, and Treatment of High Blood Pressure: the JNC VII report. JAMA 2003; 289: 2560-72.

[11] Neal B, MacMahon S, Chapman N. Blood Pressure Lowering Treatment Trialists' Collaboration. Effects of ACE inhibitors, calcium antagonists, and other blood-pressure-lowering drugs: results of prospectively designed overviews of randomised trials. Blood Pressure Lowering Treatment Trialists' Collaboration. Lancet 2000; 356: 1955-64.

[12] Sever PS, Dahlof B, Poulter NR, et al.; ASCOT investigators. Prevention of coronary and stroke events with atorvastatin in hypertensive patients who have average or lower-thanaverage cholesterol concentrations, in the Anglo-Scandinavian Cardiac Outcomes Trial-Lipid Lowering Arm (ASCOT-LLA): a multicentre randomised controlled trial. Lancet 2003; 361: 1149-58.

[13] Wolf PA, Abbott RD, Kannel WB. Atrial fibrillation as an independent risk factor for stroke: the Framingham Study. Stroke 1991; 22: 983-8.

[14] Joseph R, Han E, Tsering C, Grunfeld S, Welch KM. Platelet activity and stroke severity. J Neurol Sci 1992; 108: 1-6.

[15] Bhatt DL, Scheiman J, Abraham NS, et al. ACCF/ACG/AHA 2008 expert consensus document on reducing the gastrointestinal risks of antiplatelet therapy and NSAID use. Circulation 2008; 118: 1894909.

[16] Adams RJ, Albers G, Alberts MJ, et al. Update to the AHA/ASA recommendations for the prevention of stroke in patients with stroke and TIA. Stroke 2008; 39: 1647-52.

[17] Steering Committee of the Physicians' Health Study Research Group. Final report on the aspirin component of the ongoing Physicians' Health Study. N Engl J Med 1989; 321: 129-35.

[18] Ridker PM, Cook NR, Lee IM, et al. A randomized trial of lowdose aspirin in the primary prevention of cardiovascular disease in women. N Engl J Med 2005; 352: 1293-304.

[19] Baigent C, Blackwell L, Collins R, et al.; for the Antithrombotic Trialists' (ATT) Collaboration. Aspirin in the primary and secondary prevention of vascular disease: collaborative meta-analysis of individual participant data from randomised trials. Lancet 2009; 373: $1849-60$

[20] CAST (Chinese Acute Stroke Trial) Collaborative Group. CAST: randomised placebo-controlled trial of early aspirin use in 20,000 patients with acute ischaemic stroke. Lancet 1997; 349: 1641-9.

[21] International Stroke Trial (IST) Collaborative Group. The International Stroke Trial (IST): a randomised trial of aspirin, subcutaneous heparin, both, or neither among 19435 patients with acute ischaemic stroke. Lancet 1997; 349: 1569-81.

[22] European Stroke Organisation (ESO) Executive Committee et al. Guidelines for Management of Ischaemic Stroke and Transient Ischaemic Attack 2008, Cerebrovasc Dis 2008; 25: 457-507.

[23] Bhatt DL, Fox KA, Hacke W, et al.; CHARISMA Investigators. N Engl J Med 2006; 354: 1706-17.

[24] Sudlow CL, Mason G, Maurice JB, Wedderburn CJ, Hankey GJ. Thienopyridine derivatives $v s$. aspirin for preventing stroke and other serious vascular events in high vascular risk patients. Cochrane Database Syst Rev 2009; 4: CD001246.

[25] Johnston SC, Rothwell PM, Nguyen-Huynh MN, et al. Validation and refinement of scores to predict very early stroke risk after transient ischaemic attack. Lancet 2007; 369: 283-92.

[26] Rothwell PM, Giles MF, Chandratheva A, et al. Effect of urgent treatment of transient ischaemic attack and minor stroke on early recurrent stroke (EXPRESS study): a prospective population-based sequential comparison. Lancet 2007; 370: 1432-42.

[27] Touze E, Varenne O, Chatellier G, et al. Risk of myocardial infarction and vascular death after transient ischemic attack and ischemic stroke: a systematic review and meta-analysis. Stroke 2005; 36 : 2748-55

[28] Albers G, Amarenco P, Easton JD, Sacco RL, Teal P. Antithrombotic and thrombolytic therapy for ischemic stroke: the seventh
ACCP conference on antithrombotic and thrombolytic therapy. Chest 2004; 126: 483S-512S.

[29] Chaturvedi S. Acetylsalicylic acid + extended-release dipyridamole combination therapy for secondary stroke prevention. Clin Ther 2008; 30: 1196-205.

[30] SALT Collaborative Group. Swedish Aspirin Low-dose Trial (SALT) of $75 \mathrm{mg}$ aspirin as secondary prophylaxis after cerebrovascular ischaemic events. Lancet 1991; 338: 1345-9.

[31] The Stroke Prevention in Reversible Ischemia Trial (SPIRIT) Study Group. A randomized trial of anticoagulants $v s$. aspirin after cerebral ischemia of presumed arterial origin. Ann Neurol 1997; 42: 857-65.

[32] Mohr JP, Thompson JL, Lazar RM, et al. A comparison of warfarin and aspirin for the prevention of recurrent ischemic stroke. Warfarin-Aspirin Recurrent Stroke Study. New Engl J Med 2001; 345: 1444-51.

[33] Cambria-Kiely JA, Gandhi PJ: Aspirin resistance and genetic polymorphisms. J Thromb Thrombolysis 2002; 14: 51-8.

[34] Gum PA, Kottke-Marchant K, Welsh PA, et al. A prospective, blinded determination of the natural history of aspirin resistance among stable patients with cardiovascular disease. J Am Coll Cardiol 2003 19; 41: 961-5.

[35] Ricci S, Lewis S, Sandercock P: Previous use of aspirin and baseline stroke severity. Stroke 2006; 37: 1737-40.

[36] Bornstein NM, Karepov VG, Aronovich BD, Gorbulev AY, Treves TA, Korczyn AD. Failure of aspirin treatment after stroke. Stroke 1994; 25: 275-7.

[37] Karepov V, Tolpina G, Kuliczkowski W, Serebruany V. Plasma triglycerides as predictors of platelet responsiveness to aspirin in patients after first ischemic stroke. Cerebrovasc Dis 2008; 26: 272 6.

[38] Cha JK, Jeon HW, Kang MJ. ADP-induced platelet aggregation in acute ischemic stroke patients on aspirin therapy. Eur $\mathbf{J}$ Neurol 2008; 15: 1304-8.

[39] Hass WK, Easton ID, Adams HP Jr. Ticlopidine aspirin stroke study group (TASS). A randomized Trial comparing ticlopidine hydrochloride with aspirin for the prevention of of stroke in high risk patients. N Engl J Med 1989; 321: 501-7.

[40] Clopidogrel vs. Aspirin in Patients at Risk of Ischemic Events (CAPRIE) Steering Committee. A randomised, blinded trial of clopidogrel $v s$. aspirin in patients at risk of ischaemic events (CAPRIE). Lancet 1996; 348: 1329-39.

[41] Diener HC, Bogousslavsky PJ, Brass PLM, et al. Aspirin and lopidogrel compared with clopidogrel alone after recent ischaemic troke or transient ischaemic attack in high-risk patients MATCH): randomised, double-blind, placebo-controlled trial. Lancet 2004; 364: 331-7.

[42] Grau AJ, Reiners S, Lichy C, Buggle F, Ruf A. Platelet function under aspirin, clopidogrel, and both after ischemic stroke: a casecrossover study. Stroke 2003; 34: 849-54.

[43] Serebruany VL, Malinin AI, Ziai W, et al. Effects of clopidogrel and aspirin in combination $v s$. aspirin alone on platelet activation and major receptor expression in patients after recent ischemic stroke: for the Plavix Use for Treatment of Stroke (PLUTO-Stroke) trial. Stroke 2005; 36: 2289-92.

[44] Kennedy J, Hill MD, Ryckborst KJ, Eliasziw M, Demchuk AM, Buchan AM; FASTER Investigators. Fast assessment of stroke and transient ischaemic attack to prevent early recurrence (FASTER): a randomised controlled pilot trial. Lancet Neurol 2007; 6: 961-9.

[45] Gorelick P, Sechenova O, Hennekens CH. Evolving perspectives on clopidogrel in the treatment of ischemic stroke. J Cardiovasc Pharmacol Ther 2006; 11: 245-8.

[46] Hermann A, Dzialowski I, Koch R, Gahn G. Combined antiplatelet therapy with aspirin and clopidogrel: risk factor for thrombolysis-related intracerebral hemorrhage in acute ischemic stroke? J Neurol Sci 2009; 284: 155-7.

[47] Sibbing D, Morath G, StegheRR J, et al. Impact of proton pump inhibitors on the antiplatelet eff ects of clopidogrel. Thromb Haemost 2009; 101: 714-9.

[48] O'Donoghue MLO, Braunwald E, Antman EM, et al. Pharmacodynamic effect and clinical efficacy of clopidogrel and prasugrel with or without a proton-pump inhibitor: an analysis of two randomized trials. Lancet 2009; 374: 989-97.

[49] Shuldiner AR, O'Connell JR, Bliden KP, et al. Association of cytochrome P450 2C19 genotype with the antiplatelet effect and clinical efficacy of clopidogrel therapy. JAMA 2009; 302: 849-57. 
[50] Mega JL, Close SL, Wiviott SD, et al. Cytochrome P-450 polymorphisms and response to clopidogrel. N Engl J Med 2009; 360: 354-62.

[51] Pare G, Mehta SR, Yusuf S, et al. Effects of CYP2C19 genotype on outcomes of clopidogrel treatment. N Engl J Med 2010; 363: 1704-14.

[52] Diener HC, Cunha L, Forbes C, et al. European Stroke Prevention Study. 2. Dipyridamole and acetylsalicylic acid in the secondary prevention of stroke. J Neurol Sci 1996; 143: 1-13.

[53] The ESPRIT study group. Aspirin plus dipyridamole vs. aspirin alone after cerebral ischaemia of arterial origin (ESPRIT): randomised controlled trial. Lancet 2006; 367: 1665-73.

[54] Diener HC, Sacco RL, Yusuf S, et al.; Prevention Regimen for Effectively Avoiding Second Strokes (PRoFESS) study group. Effects of aspirin plus extended-release dipyridamole $v s$. clopidogrel and telmisartan on disability and cognitive function after recurrent stroke in patients with ischaemic stroke in the Prevention Regimen for Effectively Avoiding Second Strokes (PRoFESS) trial: a double-blind, active and placebo-controlled study. Lancet Neurol 2008; 7: 875-84.

[55] Halkes PHA, Gray LJ, Bath PMW, et al. Dipyridamole plus aspirin $v s$. aspirin alone in secondary prevention after TIA or stroke: a meta-analysis by risk. J Neurol Neurosurg Psychiatry 2008; 79: 1218-23.

[56] Berge E, Sandercock P.Anticoagulants vs. antiplatelet agents for acute ischaemic stroke. Cochrane Database Syst Rev 2002; 4 : CD003242.

[57] Paikin JS, Wright DS, Eikelboom JW. Effectiveness and safety of combined antiplatelet and anticoagulant therapy: a critical review of the evidence from randomized controlled trials. Blood Rev 2011; 25: 123-9.

[58] Yu A, Keezer MR, Zhu B, Wolfson C, Côté R Pre-Stroke Use of Antihypertensives, Antiplatelets, or Statins and Early Ischemic Stroke Outcomes. Cerebrovasc Dis 2009; 27: 398-402.
Received: ?????? 29, 2012

Revised: ????? 1, 2012

Accepted: ?????? 15, 2012 